\title{
A CLASS OF TRANSLATION PLANES OF SQUARE ORDER
}

\author{
M.L. Narayana Rao, K. Satyanarayana \\ AND G, VITHAL RAO
}

\begin{abstract}
A class of translation planes of order $p^{2 r}$, where $r$ is an odd natural number and $p$ is a prime, $p \geq 7, p \neq \pm 1(\bmod 10)$ is constructed. A salient feature shared by all these planes is that one ideal point is fixed by the translation complement and the remaining ideal points are divided into at least two orbits, one of which is of length $\mathrm{p}^{\mathrm{r}}$.
\end{abstract}

\section{Description of the class of translation planes}

This class of translation planes is constructed through 1-spread sets $[1, p .220]$ over $G F(q), q=p^{r}$, where $r$ is an odd natural number, $p$ is a prime, $p \geq 7$ and $p \neq \pm 1(\bmod 10)$. It is well-known that 5 is a nonsquare in $G F(p)$ if and only if $p \neq \pm 1(\bmod 10)$. Since $r$ is odd, 5 is a nonsquare in $\mathrm{GF}(q)$.

Let $M(a, b) \in G L(2, q)$ be defined by

$$
M(a, b)=\left(\begin{array}{cc}
a & b \\
-\frac{1}{5} b^{5} & a+b^{3}
\end{array}\right), a, b \in G F(q) .
$$

Let $C=\{M(a, b) \mid a, b \in G F(q)\}$. The set $C$ can also be written as $C=\{M(0, b)+a I \mid a, b \in G F(q)\}$, where $I$ is the $2 \times 2$ identity matrix.

It is now claimed that $C$ is a 1 -spread set over GF(q). To establish this claim, we have to verify that $L-N$ is nonsingular, for all $L, N \in C, L \neq N$. An easy computation reveals that $M(0, b)-M(0, d)$ has

Received 27 January 1984

Copyright Clearance Centre, Inc. Serial-fee code: 0004-9727/84 $\$ A 2.00+0.00$ 


$$
\lambda^{2}-\left(b^{3}-d^{3}\right) \lambda+\frac{1}{5}(b-d)^{2}\left(b^{4}+b^{3} d+b^{2} d^{2}+b d^{3}+a^{4}\right)
$$

as its characteristic polynomial. The discriminant of this polynomial is

$$
\frac{1}{5}(b-d)^{2}\left(b^{2}+3 b d+d^{2}\right)^{2}
$$

which is a nonsquare in $G F(q)$, if $b \neq d$, since 5 is a nonsquare in $G F(q)$. Thus $M(0, b)-M(0, d)$ has an irreducible characteristic polynomial for all $b, d \in G F(q), b \neq d$. Suppose $L=M(a, b), N=M(c, d)$ are two distinct matrices of $C$. Then $L-N=(a-c) I+M(0, b)-M(0, d)$. If $b=d$, then $a \neq c$ and therefore $L-N$ is nonsingular. If $b \neq d$, then $L-N$ is nonsingular, since $M(O, b)-M(0, d)$ has irreducible characteristic polynomial. This completes the proof of the claim that $C$ is a 1 -spread set over GF(q).

Let

$$
\begin{gathered}
v(M(a, b))=\{(w, x, y, z) \mid w, x \in G F(q),(y, z)=(w, x) M(a, b), \\
M(a, b) \in C\}
\end{gathered}
$$

and

$$
V(\infty)=\{(0,0, y, z) \mid y, z \in G F(q)\}
$$

be 2-dimensional subspaces of $v(4, q)$, the 4 -dimensional vector space over $G F(q)$. The incidence structure with $V(M(a, b)), M(a, b) \in C, V(\infty)$ and their cosets in the additive group of $v(4, q)$ as lines and the vectors of $\mathrm{v}(4, \mathrm{q})$ as points and inclusion as the incidence relation is the translation (affine) plane $\pi$ of order $\mathrm{q}^{2}$ associated with the 1 -spread set $C$. Since $C$ is not a ring the plane $\pi$ is nonDesarguesian. In what follows, the ideal point $V(M(a, b))$ denotes the ideal point associated with the line $V(M(a, b))$ and by a collineation, we mean a collineation of $\pi$, which belongs to the translation complement of $\pi$. It is well-known that any nonsingular lineax transformation on $V(4, q)$ induces a collineation of $\pi$ if and only if it permutes subspaces $V(M(a, b)), M(a, b) \in C$ and $V(\infty)$ among themselves. From this we get the following cxiterion:

$$
\text { A } 4 \times 4 \text { matrix }\left(\begin{array}{ll}
P & Q \\
R & S
\end{array}\right) \text {, where } P, Q, R \text { and } S \text { are } 2 \times 2 \text { matrices }
$$

over GF( $q$ ) induces a collineation of $\pi$ if and only if the following conditions are satisfied: 
(i) if $R$ is nonsingular, then $R^{-1} S \in C_{\text {; }}$ if $R$ is singular, then $R$ is the zero matrix and $S$ is nonsingular;

(ii) for $M \in C$, if $(P+M R)$ is nonsingular, then $(P+M R)^{-1}(Q+M S) \in C$;

if $(P+M R)$ is singular, then $(P+M R)$ is the zero matrix and $(Q+M S)$ is nonsingular.

The following is a useful lemma which has been used in the sequel:

LEMMA 1.1. Let $C^{\prime}$ be a (n-1)-spread set defined over $G F(q)$, where $q=p^{s}, p$ is a prime, $p>2$, and $s$ is a natural number. Let $\pi^{\prime}$ be a translation plane associated with $\mathcal{C}^{\prime}$. Further suppose that $C^{\prime}$ satisfies the condition that: if $M \in C^{\prime}$, then $-M \in \mathcal{C}^{\prime}$.

Then

(1) if there exists a collineation which fixes $V(\infty)$ and moves $V(0)$, the $n$-dimensional subspace associated with the zero matrix of $C^{\prime}$, onto $V(N)$ or moves $V(N)$ onto $V(\infty)$ and $V(\infty)$ onto $V(O)$, where $V(N) \neq V(0)$ then $M+N \in C^{\prime}$, for alz $M \in \mathcal{C}^{\prime}$;

(2) if there exists a collineation which fixes $V(O)$ and moves $V(\infty)$ onto $V(N)$ or moves $V(N)$ onto $V(0)$ and $V(0)$ onto $V(\infty)$, where $V(N) \neq V(\infty)$, then $\left(M^{-1}+N^{-1}\right)^{-1} \in C^{\prime}$, for alz nonzero matrices $M$ of $C^{\prime}$.

Proof. See [6, Lemma 3.4].

\section{Some collineations of $\pi$}

We now give some collineations of $\pi$ and indicate their actions on the ideal points. It is noticed that $N+a I \in C$, for all a $\in G F(q)$ and $-\mathrm{N} \in \mathcal{C}$ whenever $\mathrm{N} \in \mathcal{C}$. From this we obtain two collineations $\alpha(x)$ and $B$ of $\pi$, where

$$
\alpha(x)=\left(\begin{array}{ll}
I & x I \\
0 & I
\end{array}\right), \quad x \in G F(q),
$$




$$
B=\left(\begin{array}{cc}
I & 0 \\
0 & -I
\end{array}\right) \text {. }
$$

The action of $\alpha(x)$ on the set of ideal points of $\pi$ is given by:

$$
\alpha(x): V(\infty) \longrightarrow V(\infty), V(M(a, b)) \longrightarrow V(M(a+x, b)) .
$$

Let $H=\langle\alpha(x) \mid x \in G F(q)\rangle$. The collineation group $H$ is transitive on the set $\{\mathrm{V}(M(a, 0)) \mid a \in G F(q)\}$ and it is of order $q$. The action of $B$ on the set of ideal points is found to be

$$
\beta: V(\infty) \longrightarrow V(\infty), V(M(a, b)) \longrightarrow V(M(-a,-b))
$$

and it is of order 2 .

Let $\gamma$ be the mapping from $V(4, q)$ onto itself defined by:

$$
\gamma:(w, x, y, z) \longrightarrow\left(w^{p}, x^{p}, y^{p}, z^{p}\right) \text {. }
$$

This induces a collineation of $\pi$ which maps $V(M(a, b))$ onto $V\left(M\left(a^{P}, b^{p}\right)\right)$. It fixes $V(\infty), V(M(a, 0)), a \in G F(p)$ and it is of order $r$.

3. Non-existence of certain types of collineations

The aim of this section is to prove that every collineation of $\pi$ fixes $V(\infty)$. To establish this result we need the following lemmas.

LEMMA 3.1. There is no collineation which fixes $V(\infty)$ and moves $V(M(0,0))$ onto $V(M(a, b)), b \neq 0$.

Proof. By Lemma 1.1, a necessary condition for the existence of such a collineation is $N+M(a, b) \in C$, for all $N \in C$. Taking $N=M(a, b)$, we get that $2 b^{3}=(2 b)^{3}$ implying $6=0$, which is not the case.

LEMMA 3.2. There is no collineation which interchanges $V(\infty)$ and $V(M(0,0))$.

Proof. If $\delta$ is a collineation of $\pi$ interchanging $V(\infty)$ and $V(M(0,0))$ then $\delta$ is of the form $\delta=\left(\begin{array}{ll}0 & B \\ A & 0\end{array}\right)$, for some $A, B \in G L(2, q)$, satisfying the condition that for each $M \in C$ there exists $N \in C$ (and vice versa) such that

$$
A^{-1} M^{-1} B=N
$$


Taking $M=M(a, 0), a \in G F(q), a \neq 0$, we find that $a^{-1} A^{-1} B \in C$. An inspection of $C$ reveals that this can happen only if $A^{-1} B=M\left(C^{-1}, 0\right)$, for some $c \in G F(q), c \neq 0$. Now (3.1) becomes

$$
A^{-1} M^{-1} A=C N \text {. }
$$

Since the matrix $-\mathrm{N}+\mathrm{I} \in \mathrm{C}$ for all $\mathrm{N} \in \mathcal{C}$, we replace $\mathrm{N}$ by $-\mathrm{N}+\mathrm{I}$ in (3.2) and obtain the existence of a matrix $L \in C$ such that

$$
A^{-1} L^{-1} A=C(-N+I) \text {. }
$$

Adding (3.2) and (3.3) we obtain

$$
L=\left(-M^{-1}+C I\right)^{-1}
$$

The relation (3.4) implies that $\left(-M^{-1}+C I\right)^{-1} \in C$ for all $M \in C$ and $M \neq M(0,0)$. Using the fact that $-M \in C$ whenever $M \in C$ we get an equivalent statement that $\left(M^{-1}+C I\right)^{-1} \in \mathcal{C}$ for all $M \in C, M \neq M(0,0)$. Let the determinants of the matrices $M(a, b)$ and $\left(M^{-1}(a, b)+c I\right)$ be $k$ and $\ell$ respectively. After some straightforward computation we get that $k^{2} l^{2}=1$ if $\left(M^{-1}(a, b)+c I\right)^{-1} \in C$. Substituting in $k^{2} l^{2}=1$ the expressions of $k$ and $\ell$ we obtain that the determinant of $\left(c^{-1} I+M(a, b)\right)$ is $\pm c^{-2}$ for all $M(a, b) \in C$. From this it follows that the determinant of every matrix $N \in C$ is $\pm C^{-2}$. This is a contradiction since the set of matrices $\left\{M\left(-\frac{1}{2}, 1\right)+a I \mid a \in G F(q)\right\}$ contains $\frac{(q+1)}{2}$ matrices with distinct determinants. Hence the lemma.

LEMMA 3.3. There is no collineation which fixes $V(M(0,0))$ and moves $V(\infty)$.

Proof. Suppose $\delta$ is a collineation fixing $V(M(0,0))$ and moving $V(\infty)$ onto $V(M(a, b))$. Then by Lemma 1.1 , we have a necessary condition that $\left(N^{-1}+M^{-1}(a, b)\right)^{-1} \in C$, for all $N \in C, N \neq M(0,0)$. Taking $N=M(a, b), b \neq 0$, we get that $2^{-1} M(a, b) \in \mathcal{C}$. This can happen only if $6=0$, a contradiction. The case $b=0$ is dealt with as in Lemma 3.2. This completes the proof of the lemma. 
64 M.L. Narayana Rao, K. Satyanarayana and G. Vithal Rao

LEMMA 3.4. There is no colzineation which sends $V(M(a, b))$ onto $V(\infty)$ and $V(\infty)$ onto $V(M(0,0)),(a, b) \neq(0,0)$.

Proof. Suppose $\sigma$ is a collineation mapping $V(M(a, b))$ onto $V(\infty)$ and $V(\infty)$ onto $V(M(0,0)),(a, b) \neq(0,0)$. Then by Lemma 1.1 we obtain a necessary condition that $N+M(a, b) \in C$, for all $N \in C$. Taking $b \neq 0$ and $N=M(a, b)$ we get that $2 M(a, b) \in C$, which is $a$ contradiction. Suppose $b=0$. Then the collineation $\sigma^{-1} \alpha(x) \sigma$ fixes $V(M(0,0))$ and moves $V(\infty)$, a contradiction to Lemma 3.3. Thus the lemma is proved.

Using Lemmas $3.1-3.4$ we conclude that the ideal points corresponding to $V(M(0,0))$ and $V(\infty)$ are not in the same orbit.

THEOREM 3.5. Every collineation of $\pi$ fixes $V(\infty)$.

Proof. Suppose $\delta$ is a collineation of $\pi$ which moves $V(\infty)$. Then by Lemma $3.3, \delta$ moves $V(M(0,0))$. Let the images of $V(\infty)$, $V(M(0,0))$ and $V(M(1,0))$ under $\delta$ be $V(M(a, b)), V(M(c, d))$ and $\mathrm{V}(\mathrm{M}(\mathrm{e}, \mathrm{f}))$ respectively. By Proposition $6[4, \mathrm{p} .491]$ we get that, for each $M \in C$ there exists $N \in C$ (and vice versa) such that

$$
\left[(M-M(a, b))^{-1}-(M(c, d)-M(a, b))^{-1}\right]\left[(M(e, f)-M(a, b))^{-1}-(M(c, d)-M(a, b))^{-1}\right]^{-1}
$$$$
=A^{-1} \mathrm{NA}
$$

for some $A \in G L(2, q)$. Replacing $N$ by $-N$ in (3.5) we get the existence of a matrix $L$ in $C$ such that

$$
\left[(L-M(a, b))^{-1}-(M(c, d)-M(a, b))^{-1}\right]\left[(M(e, f)-M(a, b))^{-1}-(M(c, d)-M(a, b))^{-1}\right]^{-1}
$$

$$
=A^{-1}(-N) A \text {. }
$$

On adding (3.5) and (3.6) and simplifying we get that for each matrix $M \in \mathcal{C}$ there exists a matrix $L \in C$ such that

$$
\text { (3.7) }(L-M(a, b))^{-1}+(M-M(a, b))^{-1}=2(M(c, d)-M(a, b))^{-1} \text {. }
$$

The relation (3.7) implies that the mapping

$$
M \longrightarrow M(a, b)+\left[-(M-M(a, b))^{-1}+2(M(c, d)-M(a, b))^{-1}\right]^{-1}
$$


is a collineation of $\pi$. The collineation given by (3.8) maps $V(\infty)$ onto $V(Q)$ where $Q=2^{-1}(M(a, b)+M(c, d)) \in C$. A necessary condition for $Q$ to be in $C$ is that $b= \pm d$. This implies that $\delta$ moves $V(\infty)$ onto $V(M(a, b))$ and $V(M(0,0))$ onto $V(M(c, \pm b))$. But the ideal points $V(M(a, b))$ and $V(M(c, \pm b))$ are in the same orbit under $(\beta, H)$. This forces $V(\infty)$ and $V(M(0,0))$ to be in the same orbit, a contradiction. Hence the lemma.

4. The translation complement of $\pi$

It may be observed from sections 2 and 3 that the translation complement fixes $V(\infty)$ and is transitive on $\{v(M(a, 0)) \mid a \in G F(q)\}$. Let $J$ be the group of all collineations of $\pi$ that fix $V(\infty)$ and $V(M(0,0))$. A coset decomposition of the translation complement $G$ is given by

$$
\mathrm{a \in GF}(\mathrm{q}){ }^{J \mathrm{x}} \mathrm{a}
$$

where $x_{a}$ is a collineation fixing $V(\infty)$ and mapping $V(M(0,0))$ onto $V(M(a, 0))$. We may take $x_{a}$ to be $\alpha(a)$ and $G=\langle J, H\rangle$. It is seen that $J \supset\langle\gamma, \beta\rangle$. Using Theorem 3.5 and Lemma 3.1 we get that $G$ divides the set of ideal points into at least three orbits, one containing a single ideal point and the second containing $q$ ideal points. This orbit structure makes the plane $\pi$ distinct from the following known square order planes:
(a) the generalized André planes (including Hall planes)
(b) the flag transitive planes
[5],
(c) the planes of Hering of order $q^{2},(q \equiv-1(\bmod 6))$
[3],
(d) the planes of walker of order $q^{2},(q \equiv-1(\bmod 6))$

Further, the present construction defines planes for some primes covered in (c) and (d), and some other primes not covered in (c) and (d).

One of the authors [7] computed $G$ in the particular case $q=7$ and established that $G$ actually divides the set of ideal points into 3 orbits of lengths 1,7 and 42 and $G$ is of order 2016. 
66 M.L. Narayana Rao, K. Satyanarayana and G. Vithal Rao

\section{References}

[1] P. Dembowski, Finite Geometries (Springer-Verlag, Berlin, 1968).

[2] D.A. Foulser, "A generalization of Andre's systems", Math. Z. 100 (1967), 380-395.

[3] C. Hering, "A new class of quasifields", Math. 2. 118 (1970), 56-57.

[4] D.M. Maduram, "Matrix representation of translation planes", Geom. Dedicata, 4 (1975), 485-492.

[5] M.L. Narayana Rao, "A class of flag transitive planes", Proc. Amer. Math. Soc. , 39 (1973), 51-56.

[6] M.L. Narayana Rao and K. Satyanarayana, "A new class of square order planes", J. Combin. Theory Ser. A, 35 (1983), 33-42.

[7] K. Satyanarayana, "On some translation planes of square and cube orders and their translation complements", (PhD Dissertation, Osmania University, India, 1982).

[8] M. Walker, "A class of translation planes", Geom. Dedicata 5 (1976), 135-146.

Department of Mathematics,

Osmania University,

Hyderabad-500 007,

India 